\title{
Peroral video cholangioscopic findings in a biliary intraductal mucinous cystic neoplasm
}
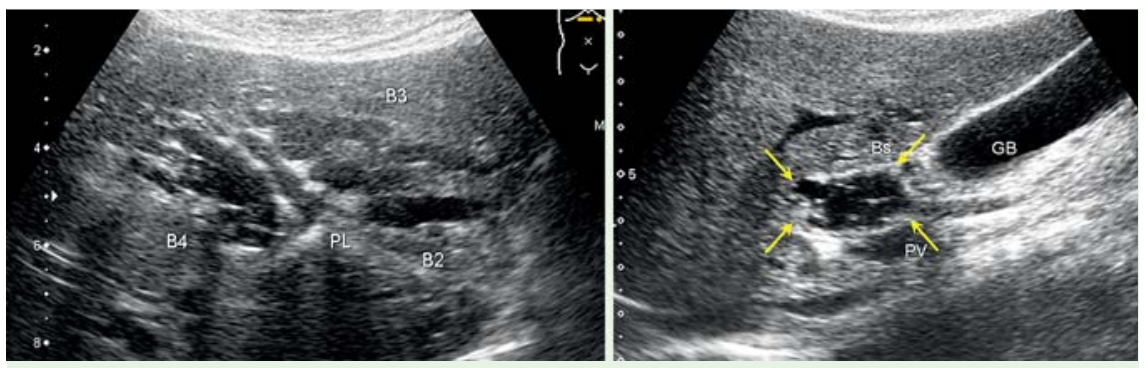

Fig. 1 Ultrasound images showing dilatation of $\mathrm{B}_{2}, \mathrm{~B}_{3}, \mathrm{~B}_{4}$, and multilocular perihilar cystic lesions (arrows). PL, left portal vein; Bs, perihilar bile duct; $G B$, gallbladder; PV, portal vein.
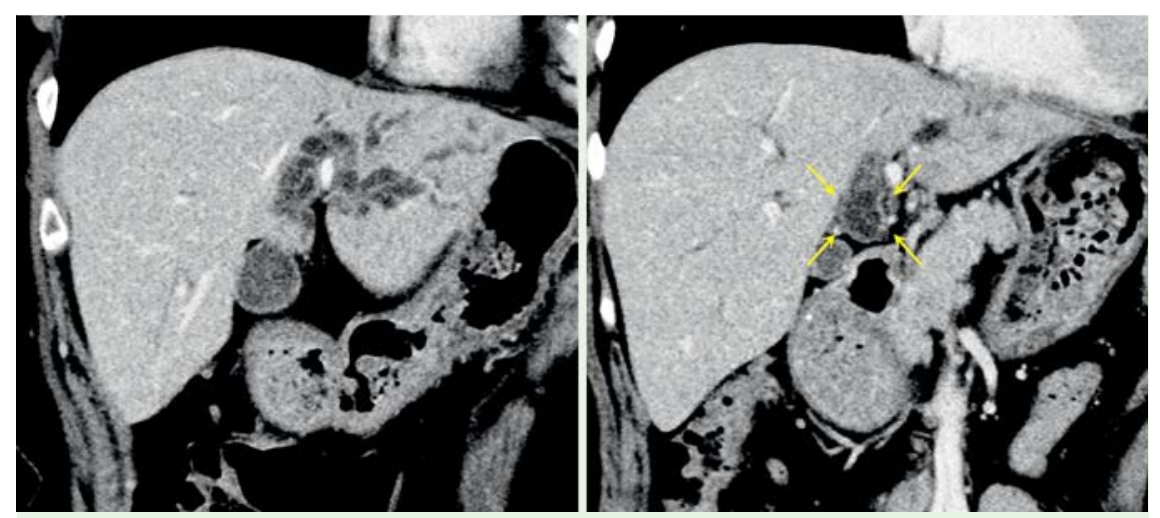

Fig. 2 Contrast-enhanced computed tomography images showing multilocular perihilar cystic lesions (arrows) with dilatation of $\mathrm{B}_{2}, \mathrm{~B}_{3}$, and $\mathrm{B}_{4}$.
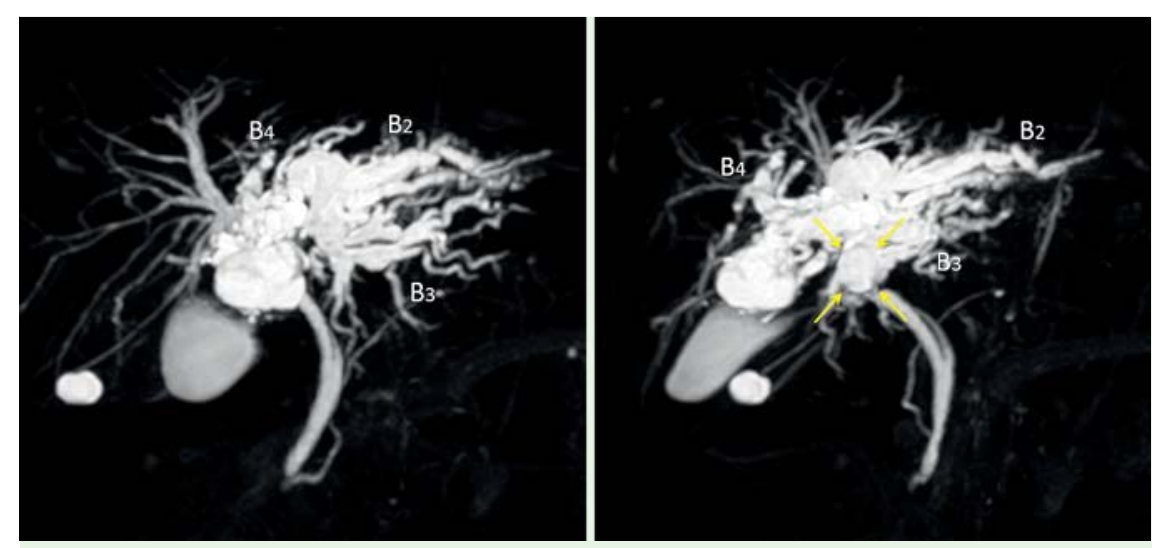

Fig. 3 Magnetic resonance cholangiopancreatography images showing multilocular perihilar cystic lesions (arrows) with dilatation of $\mathrm{B}_{2}, \mathrm{~B}_{3}$, and $\mathrm{B}_{4}$.

Mucinous cystic neoplasm (MCN) of the liver is a biliary counterpart of pancreatic MCN, usually occurring in the liver, but occasionally extending to the extrahepatic bile duct $[1,2]$. We present a case of MCN with extrahepatic bile duct extension diagnosed by peroral video cholangioscopy (POVCS).
A 49-year-old woman was admitted with a week of vomiting without abdominal pain, and jaundice. Abdominal ultrasound ( $\bullet$ Fig. 1), contrast-enhanced computed tomography ( $\bullet$ Fig.2), and magnetic resonance cholangiopancreatography ( $\odot$ Fig.3) revealed a multilocular perihilar cystic lesion with dilation of $\mathrm{B}_{2}$,
$\mathrm{B}_{3}$, and $\mathrm{B}_{4}$. Endoscopic retrograde cholangiography showed an elliptical defect from the left hepatic duct to the perihilar bile duct ( $\bullet$ Fig.4). Intraductal ultrasonography demonstrated multilocular, cyst-in-cyst structures in the extrahepatic bile duct ( $\bullet$ Fig.5). Subsequent POVCS (CHF-B260; Olympus, Tokyo, Japan) revealed a mass filling the bile duct covered with smooth and translucent bile duct mucosa, suggesting a subepithelial growth without bile duct communication ( $\bullet$ Fig. 6; $\bullet$ Video 1). A directly visualized biopsy showed no evidence of malignancy. We diagnosed intraductal MCN of the liver based on the findings of the imaging modalities. The patient underwent left lobectomy and caudate lobectomy. A cholangiogram of the resected specimen showed no biliary communication. Histologically, the dilated ducts were filled with a polypoid cystic mass consisting of lining columnar epithelium and ovarianlike stroma, features consistent with MCN with low grade dysplasia. The tumor was located beneath the lining epithelium of the left hepatic duct and there was no communication between the ductal lumen and cystic spaces. There was no evidence of recurrence at 3-year follow-up. This is the first report of biliary intraductal MCN, suggesting an origin from Glisson's capsule of the large hepatic bile duct. A recent report was the first to describe extrahepatic bile duct intraductal MCN using POVCS [3]. A cystic mass with smooth surface and compression due to distension without bile duct communication may be the characteristic findings of biliary intraductal MCN with POVCS.

\section{Endoscopy_UCTN_Code_CCL_1AZ_2AC}

\section{Competing interests: None}

\section{Hiroshi Kawakami ${ }^{1,2}$, Yoshimasa Kubota',2, Kimitoshi Kubo²,}

Daisuke Sato ${ }^{3,4}$, Tomoko Mitsuhashi' ${ }^{3}$, Satoshi Hirano ${ }^{4}$, Naoya Sakamoto ${ }^{2,5}$

${ }^{1}$ Department of Gastroenterology and Hepatology, Center for Digestive Disease, The University of Miyazaki, Miyazaki, Japan

2 Department of Gastroenterology and Hepatology, Hokkaido University Hospital, Sapporo, Japan

3 Department of Surgical Pathology, Hokkaido University Hospital, Sapporo, Japan ${ }^{4}$ Department of Gastroenterological Surgery II, Hokkaido University Graduate School of Medicine, Sapporo, Japan 


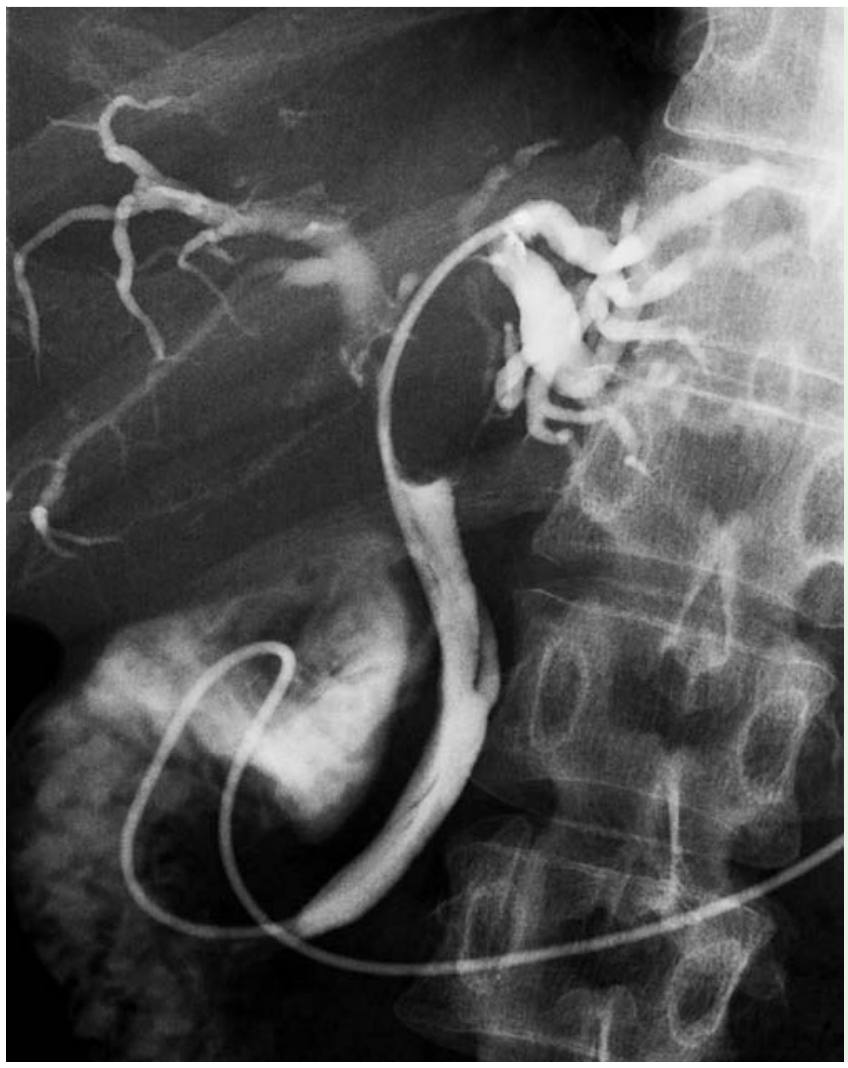

Fig.4 Endoscopic retrograde cholangiography showing an elliptical defect from the left hepatic duct to the perihilar bile duct.

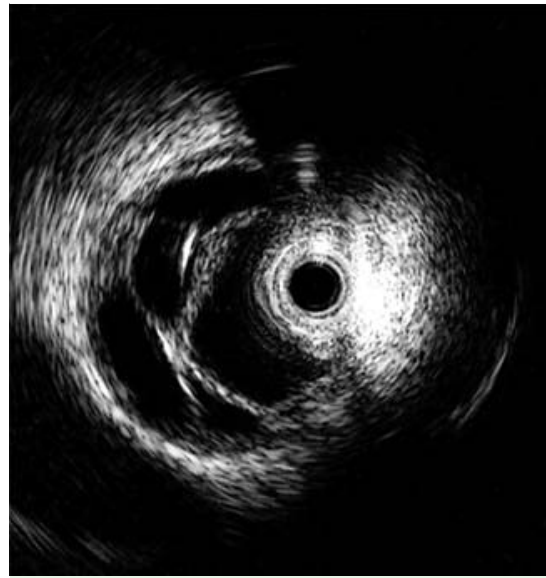

Fig. 5 Intraductal ultrasonography showing multilocular, cyst-in-cyst structures in the extrahepatic bile duct.

${ }^{5}$ Department of Gastroenterology and Hepatology, Hokkaido University Graduate School of Medicine, Sapporo, Japan

\section{Acknowledgments \\ $\nabla$}

We express our deepest appreciation to Dr. Taiki Kudo, Dr. Yoko Taya, Dr. Shuhei Kawahata (Department of Gastroenterology and Hepatology, Hokkaido University Hospital, Sapporo, Japan), Dr. Kazumichi

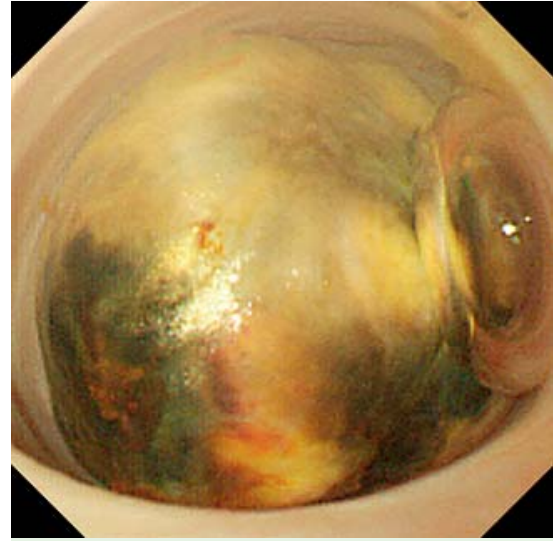

Fig. 6 Endoscopic image showing the smooth and hyperpermeable bile duct mucosa, suggesting intraductal growth.

Kawakubo (Department of Gastroenterology and Hepatology, Hokkaido University Graduate School of Medicine, Sapporo, Japan), and Dr. Masaki Kuwatani (Division of Endoscopy, Hokkaido University Hospital, Sapporo, Japan) for clinical advice. We also thank Dr. Yuko Omori (Department of Pathology, Teine-Keijinkai Hospital, Sapporo, Japan) for skillful analysis of the case pathology, Dr. Yoh Zen (Department of Diagnostic Pathology, Kobe University Graduate School of Medicine), and Dr.

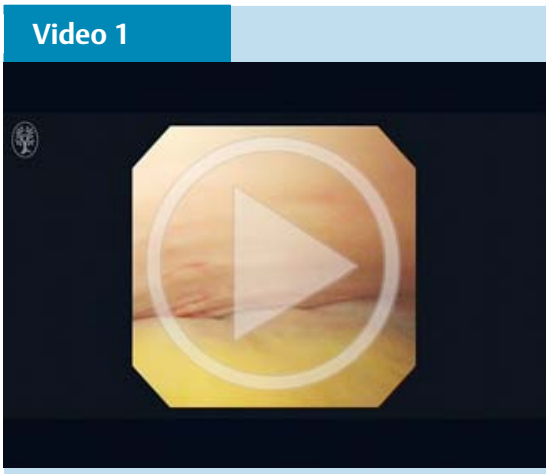

Peroral video cholangioscopy (CHF-B260, Olympus, Tokyo, Japan) performed using a mother-baby system under fluoroscopic guidance.

Yoshihiro Matsuno (Department of Surgical Pathology, Hokkaido University Hospital, Sapporo, Japan) for professional advice on the case pathology.

\section{References}

1 Nakanuma Y, Curabo MP, Franceschi S et al Intrahepatic cholangiocarcinoma. In: Hamilton SR, Aaltonen LA, eds. World Health Organization Classification of Tumors. Pathology and Genetics of Tumours of the Digestive System. Lyon: IARC Press; 2010: $217-$ 224

2 Zen $Y$, Jang KT, Ahn $S$ et al. Intraductal papillary neoplasms and mucinous cystic neoplasms of the hepatobiliary system: demographic differences between Asian and Western populations, and comparison with pancreatic counterparts. Histopathology 2014; 65: 164-173

3 Watanabe S, Sato T, Hosono Ket al. Diagnosis of biliary cystadenoma by peroral video cholangioscopy. Endoscopy 2013; 45: E284-E285

\section{Bibliography}

DOI http://dx.doi.org/

10.1055/s-0042-104654

Endoscopy 2016; 48: E152-E153

(c) Georg Thieme Verlag KG

Stuttgart · New York

ISSN 0013-726X

\section{Corresponding author}

\section{Hiroshi Kawakami, MD, PhD}

Department of Gastroenterology and Hepatology Center for Digestive Disease

The University of Miyazaki

5200, Kihara, Kiyotake-cho, Miyazaki

Miyazaki 889-1692

Japan

Fax: +81-985-85-9802

hiropon@med.miyazaki-u.ac.jp 